\section{PTU-065 PROTON PUMP INHIBITORS AND FRACTURE RISK - A BONE OF CONTENTION AMONG UK GASTROENTEROLOGISTS?}

${ }^{1}$ Luke Materacki*, ${ }^{2}$ Benjamin Colleypriest. ${ }^{1}$ Southmead Hospital, Bristol, UK; ${ }^{2}$ Royal United Hospital, Bath, UK

\subsection{6/gutjnl-2019-BSGAbstracts.281}

Introduction Proton pump inhibitors (PPIs) are one of the most commonly prescribed drugs worldwide and their use is often prolonged without clear indication. ${ }^{1}$ Although PPIs are widely regarded as safe, meta-analyses of observational studies has suggested PPI use significantly increases fracture-risk. ${ }^{2,3}$ Current guidelines recommend that people over the age of 50 taking PPIs may be considered for fracture-risk assessment, particularly in the presence of other risk factors. ${ }^{4}$

This study aimed to assess the attitudes of UK gastroenterologists towards fracture-risk assessment in PPI users over the age of 50 .

Methods A survey of UK gastroenterology consultants was conducted between 17/02/2018 and 17/03/2018 using the Survey Monkey website.

Results Of the 65 UK gastroenterologists who responded, $53.9 \%$ believed that PPI use increased fracture-risk. In PPI users aged over 50 years, gastroenterologists rarely $(52.5 \%)$ or never $(42.5 \%)$ assessed fracture-risk and infrequently delegated fracture-risk assessment to GPs, rarely or never in $40.0 \%$ and $57.5 \%$ of cases respectively. All gastroenterologists felt that GPs should take responsibility for assessing fracture-risk in this patient group with roughly half accepting co-responsibility.

$40.0 \%$ of gastroenterologists were unaware that a 10 -year fracture-risk score above $10 \%$ should prompt a bone densitometry (DEXA) scan in PPI users aged over 50. The majority of gastroenterologists had not evaluated fracture-risk with a Qfracture or FRAX score $(80.0 \%)$ or DEXA scan $(67.5 \%)$ in the preceding year.

Conclusions This study suggests that roughly half of UK gastroenterologists fail to recognise the association between PPI use and increased fracture-risk. Most gastroenterologists neglect fracture-risk assessment in PPI users aged over 50 and only half believe this should be their responsibility despite commonly encountering these patients. There is a need to educate gastroenterologists about the fracture-risk in PPI users aged over 50 .

\section{REFERENCES}

1. Haastrup PF, Rasmussen S, Hansen JM, et al. General practice variation when initiating long-term prescribing of proton pump inhibitors: a nationwide cohort study. BMC Fam Pract 2016;17:57.

2. Eom CS, Park SM, Myung Sk, Yun jM, Ahn jS. Use of acid-suppressive drugs and risk of fracture: a meta-analysis of observational studies. Ann Fam Med 2011;9 (3):257-67.

3. Kwok CS, Yeong JK, Loke YK. Meta-analysis: risk of fractures with acid-suppressing medication. Bone 2011;48(4):768-76.

4. SIGN 142 Management of osteoporosis and the prevention of fragility fractures, a national clinical guideline. March 2015. Accessible by URL: https://www.sign.ac uk/assets/sign142.pdf (accessed 12/02/2019)

\section{PTU-066 MANAGEMENT OF HELICOBACTER PYLORI INFECTION IN PATIENTS WITH UPPER GI BLEEDING: COMPLIANCE WITH GUIDELINES}

${ }^{1}$ Luke Sullivan*, ${ }^{2}$ Nekisa Zakeri, ${ }^{2}$ Rupert Negus, ${ }^{1}$ Marsha Y Morgan. ${ }^{1}$ UCL Institute for Liver and Digestive Health, University College London, London, UK; ${ }^{2}$ Royal Free Hospital, London, UK

\subsection{6/gutjnl-2019-BSGAbstracts.282}

Introduction Acute upper gastrointestinal bleeding (AUGIB) is a common complication of peptic ulcer disease (PUD) defined as not only peptic ulceration but also erosive gastritis and duodenitis, Helicobacter pylori (H. pylori) infection is the major cause of PUD. NICE guidelines recommend that patients identified with PUD should be tested and treated for $H$ pylori if infected. Post-eradication testing is mandated to confirm successful eradication. The aim of this study was to determine compliance with national guidelines for the management of $H$. pylori in those with PUD-associated AUGIB.

Methods Retrospective data were collected on all patients presenting to the Royal Free Hospital, London, with non-variceal AUGIB between 1 January and 31 December 2017. Prospective data were collected between 1 February and 1 April 2018. Compliance with guidelines was judged using predetermined criteria and classified as: poor (<67\% compliance); moderate (67-75\%); good (>75\%); or excellent $(>90 \%)$.

Results A total of 203 patients presented with non-variceal AUGIB during 2017, of whom 148 underwent endoscopy. Sixty-seven of those endoscoped met criteria for $H$. pylori testing but only $35(52.2 \%)$ were investigated despite an absence of active bleeding or high-risk stigmata (compliance poor) (table 1). Of the 32 cases not tested, 22 exhibited overt ulceration at endoscopy, with the remaining cases demonstrating erosive changes. Four of the 55 non-endoscoped patients were tested for $H$ pylori by other means. Overall, 15 (38.5\%) of the 39 patients investigated tested positive for $H$ pylori; 14 (93.3\%) were prescribed eradication therapy (compliance excellent) while a further five patients were treated empirically. Of the 19 treated patients, only nine (47.4\%) underwent post-eradication testing (compliance poor); all however tested negative. Prospective compliance rates in 36 patients exhibited similar poor compliance although samples were small (table 1).

Abstract PTU-066 Table 1 Management of $H$. pylori and compliance with associated NICE guidelines

\begin{tabular}{|c|c|c|c|c|c|}
\hline $\begin{array}{l}\text { Management } \\
\text { Step }\end{array}$ & NICE Guideline & Study & $\begin{array}{l}\text { Eligible } \\
\text { (n) }\end{array}$ & $\begin{array}{l}\text { Performed } \\
\text { (n) }\end{array}$ & $\begin{array}{l}\text { Compliance } \\
(\%)\end{array}$ \\
\hline Endoscopic & Test for H. pylori if & $\mathrm{R}$ & 67 & 35 & Poor $(52.2 \%)$ \\
\hline detection & evidence of PUD & $P$ & 9 & 5 & Poor $(55.6 \%)$ \\
\hline \multirow[t]{2}{*}{ Eradication therapy } & $\begin{array}{l}\text { Offer those testing } \\
\text { positive }\end{array}$ & $\mathrm{R}$ & 15 & 14 & $\begin{array}{l}\text { Excellent } \\
(93.3 \%)\end{array}$ \\
\hline & eradication therapy & $P$ & 3 & 2 & Poor $(66.7 \%)$ \\
\hline Post-Eradication & Re-test using a $\mathrm{C}^{13}$ & $\mathrm{R}$ & 19 & 8 & Poor $(42.1 \%)$ \\
\hline Testing & urea breath test. & $P$ & 2 & 0 & Poor $(0 \%)$ \\
\hline
\end{tabular}


Conclusion Approximately 50\% of patients presenting with AUGIB with endoscopic features of PUD were not tested for H. pylori. Almost $40 \%$ of those who were tested were infected. Post-eradication investigation rates were particularly low. No clear explanation for this level of non-compliance with guidelines is apparent but enforcement is clearly warranted.

\section{PTU-067 HELICOBACTER PYLORI RESISTANCE: A COMPLEX SOCIOECONOMIC PROBLEM?}

${ }^{1}$ Luke Sullivan*, ${ }^{2}$ Nekisa Zakeri, ${ }^{2}$ Rupert Negus, ${ }^{2}$ Damien Mack, ${ }^{1}$ Marsha Y Morgan. ${ }^{1}$ UCL Institute for Liver and Digestive Health, University College London, London, UK; ${ }^{2}$ Royal Free Hospital, London, UK

\subsection{6/gutjnl-2019-BSGAbstracts.283}

Introduction Helicobacter pylori $(H$. pylori) infection is the primary cause of peptic ulcer disease and is globally prevalent. Although eradication therapy is effective, emerging antibiotic resistance rates are of considerable concern. H. pylori infection is more prominent in deprived populations but the effects of ethnicity and social determinants of health on $H$. pylori resistance is unclear. The aim of this study was to establish the significance of socioeconomic and ethnic influences on $H$. pylori resistance.

Methods The study population comprised of patients with recurrent/previous $H$. pylori infection plus current dyspeptic symptoms referred to the Royal Free Hospital, London, from 1 January 2017 to 1 September 2018, for endoscopic investigations. Gastro/duodenal biopsy samples were sent for culture and sensitivity testing. Demographic and clinical data were collected. Self-reported ethnic origin was recorded. Deprivation was analysed on a postcode basis, using the 2015 English Indices of Deprivation, by means of average decile ranking (1-10). Ethnic and socioeconomic differences between patients exhibiting resistance and those fully sensitive/culture negative were explored.

Results A total of 107 patients, including 26 children, (61.7\% female; median (range) age 37.5 (4-81) years; 43.9\% Caucasian, 39.2\% Asian), were sampled. Five were excluded (3 contaminated, 2 inappropriate samples). Forty of the remaining 102 samples yielded positive cultures; of these 6/40 were fully sensitive but 34 (33\% of total sample) displayed antibiotic resistance, most commonly to metronidazole (91.2\%), and/or clarithromycin (77.4\%). Dual resistance was present in $52.9 \%$. Resistance to amoxicillin $(n=2)$ and levofloxacin $(n=3)$ was also identified. The remaining 62 samples were culture negative. There were no differences in the age or sex distributions between the two cohorts. Likewise, there was no significant difference in the ethnic distribution between patients displaying antibiotic resistance and those sensitive/culture negative (Asian $47 \%$ vs. $35 \% \mathrm{Chi}^{2} \mathrm{P}=0.25$ ) (figure $1 \mathrm{~A}$ ). Deprivation analysis showed very similar average decile rankings in both patient groups although distribution was skewed to the deprived end of each domain in both (figure 1B).

Conclusion One-third of biopsy samples referred for sensitivity testing, during the study period, exhibited antibiotic resistance, most commonly to first and second line eradication therapies. No significant differences were observed in ethnicity or in deprivation between patients exhibiting resistance and those sensitive/culture negative. However, trends identified in this study need to be further explored.
Abstract PTU-067 Figure 1 Comparative analysis of resistant vs. sensitive/no culture cohorts. (A) Ethnicity (B) Deprivation

\section{PTU-068 UPPER GASTROINTESTINAL BLEEDING IN THE PRESENCE OR ABSENCE OF PORTAL HYPERTENSION AND/OR NON-VARICEAL LESIONS}

\author{
${ }^{1,2}$ Ali Taha*, 'Matthew Friar, ${ }^{1}$ Caroline McCloskey, ${ }^{1}$ Theresa Craigen, ${ }^{2}$ Wilson Angerson. \\ ${ }^{1}$ University Hospital Crosshouse, Kilmarnock, Kilmarnock, UK; ${ }^{2}$ University of Glasgow \\ Medical School, Glasgow, UK
}

\subsection{6/gutjn|-2019-BSGAbstracts.284}

Introduction Upper gastrointestinal bleeding (UGIB) is caused by variceal lesions related to portal hypertension (PHT), or non-variceal lesions (NVL). Some patients may present with both PHT and NVL. We aimed to study the outcomes and possible interaction between the two conditions with adjustment for age, sex, smoking and alcohol.

Methods Patients presenting with UGIB were classified according to the presence or absence of PHT or NVL, or both. PHT included varices in the oesophagus or stomach and gastropathy or duodenopathy. Other lesions were considered as non-variceal, such as erosive oesophagitis, peptic ulcers, erosive gastritis or duodenitis, etc.

Logistic regression was used to assess PHT and NVL as predictive factors for UGIB outcomes, adjusting for demographic variables and testing for an interaction between PHT and NVL.

Results Between 2008-2016, the following subgroups entered the analysis: No PHT/NVL $(\mathrm{n}=595,56 \%$ males, median age 64 years); NVL only ( $n=1556,63 \%$ males, age 67); PHT only $(n=187,63 \%$ males, age 56); and PHT + NVL $(n=106$, $65 \%$ males, age 58). The results of the logistic regression analysis are shown in table 1.

Abstract PTU-068 Table 1 Odds ratios (OR) with 95\% confidence intervals $(\mathrm{Cl})$ for the outcomes of UGI bleeding in patients with portal hypertension (PHT) and/or non-variceal lesions (NVL). The reference group $(\mathrm{OR}=1.0)$ is those without PHT or NVL

\begin{tabular}{|c|c|c|c|c|c|}
\hline Outcome & $\begin{array}{l}\text { Endoscopic } \\
\text { findings }\end{array}$ & $\begin{array}{l}\text { OR }(95 \% \\
\mathrm{Cl}) \\
\text { Unadjusted }\end{array}$ & $P$ value & $\begin{array}{l}\text { OR (95\% Cl) } \\
\text { Adjusted for age, sex, } \\
\text { smoking, alcohol }\end{array}$ & $\begin{array}{l}P \\
\text { value }\end{array}$ \\
\hline Admitted & PHT & 2.25 (1.73- & $<0.001$ & $2.58(1.90-3.52)$ & $<0.001$ \\
\hline$>7$ days & NVL & $\begin{array}{l}2.91) \\
1.51(1.24- \\
1.84)\end{array}$ & $<0.001$ & $1.37(1.11-1.69)$ & 0.003 \\
\hline Transfused & $\begin{array}{l}\text { PHT } \\
\text { NVL no PHT } \\
\text { NVL with } \\
\text { PHT }\end{array}$ & $\begin{array}{l}5.28(3.71- \\
7.51) \\
1.95(1.56- \\
2.45) \\
0.61(0.38- \\
0.98)\end{array}$ & $\begin{array}{l}<0.001 \\
<0.001 \\
0.043\end{array}$ & $\begin{array}{l}6.56(4.43-9.71) \\
1.83(1.44-2.32) \\
0.58(0.35-0.95)\end{array}$ & $\begin{array}{l}<0.001 \\
<0.001 \\
0.031\end{array}$ \\
\hline Died & PHT & 5.17 (3.36- & $<0.001$ & $6.09(3.58-10.37)$ & $<0.00$ \\
\hline$\leq 30$ days & NVL & $\begin{array}{l}7.96) \\
2.02(1.29- \\
3.15)\end{array}$ & 0.002 & $1.94(1.23-3.07)$ & 0.005 \\
\hline
\end{tabular}

